\title{
A New Conjugate Gradient Algorithm using Kafaki and Gambry Coefficient in Unconstrained Optimization
}

\author{
Sawsan Sami Ismael \\ Department of Mathematics, \\ College of Education of Pure Science \\ University of Mosul, Mosul, IRAQ- \\ Sawsan.sami14@uomosul.edu.iq
}

DOI: $\underline{10.33899 / \text { edusj.2019.162953 }}$

\section{Received 27 / 11 / 2018}

\author{
Accepted \\ 21 / 01 / 2019
}

\begin{abstract}
:
In this paper, a new conjugate gradient formula using Kafaki and Gambry is proposed. And we give the global convergence of new method.

Also we give descent and sufficient descent conditions.

Numerical results, show that this new formula is more efficient for solving unconstrained optimization problems comparing with other method (H/S),depending on the iterations and the number of functions evaluation.
\end{abstract}

Keywords: unrestricted optimization, conjugation, gradient conditions, adequate regression, and correlation requirement. 


\title{
خوارزمية جديدة للتدرج المترافق \\ باستخدام معامل كافاكي وغانبري في الامثلية غير المقيدة
}

\author{
سوسن سامي اسماعيل \\ قسم الرياضيات / كلية التربية للعلوم الصرفة سئة \\ جامعة الموصل، الموصل ، العراق \\ Sawsan.sami14@uomosul.edu.iq
}

DOI: $\underline{10.33899 / \text { edusj.2019.162953 }}$

القبول

2019 / 01 / 21
الاستلام

2018 / 11 / 27

الملخص

في هـا البحث تم اقتراح صيغة جديدة للمتجهات المترافقة باسـتعمال معامل كـافـاكي و غانبري.

الخوارزمية الجديدة تحقق التقارب الثامل وكذلك شروط الانحدار والانحدار الكافي. النتائج العددية اظهرت ان الخوارزمية الجديدة ذات كفاءة جيدة في حل مسائل الامثلية غير المقيدة مقارنة مع الخوارزمية الاصلية (H/S) بالاعتماد على عدد التكرارات وعدد حساب الدالة.

الكلمات المفتاحية: الامثلية غير المقيدة، التدرج المترافق، شروط الانحدار والانحدار الكافي وشرط الترافق.

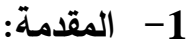
ليكن لاينا مسألة الامثلية غير المقيدة الآتية:

$\min _{x \in R^{n}} f(x)$

إذ ان ${ }^{\prime}$ ان $f: R^{n} \rightarrow$ هي دالة مستمرة وقابلة للاشتقاق، ومتجه التدرج لها موجود وهو

، (n) كبير اذ يبدأ الحل من العلاقة التكرارية التي يمكن الحصول عليها من:

$x_{i+1}=x_{i}+\alpha_{i} s_{i} \quad i=0,1,2, \ldots$

$s_{i+1}=\left\{\begin{array}{cc}-g_{i} & \text { if } i=0 \\ -g_{i+1}-\beta_{i} s_{i} & \text { if } i \geq 1\end{array}\right.$

إذ ان 00 > $\alpha_{i}$ طول الخطوة، و $s_{i}$ اتجاه البحث، ويعطى بالصيغة الاتية:

معامل الترافق لخوارزمية التدرج هو

هناك خوارزميات كثيرة تستخدم لحل مسائل (1-1) ومن اهمها التدرج المترافق المعروفة تتضمن معاملات الترافق

Dai-Liao (DL), Dai-Yuan (DY), Fletcher-Reeves (FR), Conjugate Descent (CD), Hestenes-Stiefel (HS), Polak-Ribiere-Polyak (PRP) and Liu-Storey (LS) $([1],[3],[4],[5],[7],[9],[11],[12])$. 
ومن اهم الامور التي يجب التركيز عليها في هذه الخوارزميات وغيرها من خوارزميات مسائل الامثلية غير المقيدة هو التقارب باتجاه الحل المضبوط والذي يضمن الحصول على حلول تقريبية وبعدد خطوات اقل.

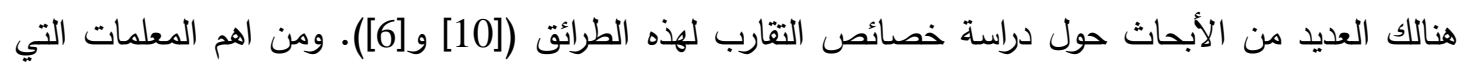
يعتمد عليها التقارب لأي طريقة هو كيفية حساب حجم الخطوة $\alpha_{i}$ والذي يحسب بعدة طرق الابت دمها شروط $f\left(x_{i}+\alpha_{i} s_{i}\right)-f\left(x_{i}\right) \leq \rho \alpha_{i} g_{i}^{T} s_{i}$ Wolfe $\left|g\left(x_{i}+\alpha_{i} s_{i}\right)^{T} s_{i}\right| \leq-\sigma g_{i}^{T} s_{i}$ إذ ان

اضافة الى ان هنالك العديد من الخوارزميات (على سبيل المثال: طريقة الانحدار الثديد Steepest descent method وطرائق أشباه نيوتن Quasi-Newton methods) لحل مسائل الأمثلية غير المقيدة $f\left(x_{i}+\alpha_{i} s_{i}\right)-f\left(x_{i}\right) \leq \rho \alpha_{i} g_{i}^{T} s_{i}$ والتي تم برهنة تقاربها استناداً على شروط Wolfe الاصلية [13] و [14]: $g\left(x_{i}+\alpha_{i} s_{i}\right)^{T} s_{i} \geq \sigma g_{i}^{T} s_{i}$

تم تقسيم البحث على النحو التالي: في الجزء الاول مقدمة عن المتجهات المترافقة وفي الجزء الثاني

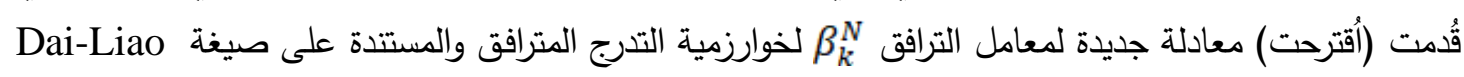
$\beta_{i}^{(D L)}=\frac{g_{i+1}^{T}\left(y_{i}-t v_{i}\right)}{s_{i}^{T} y_{i}} \quad ; t>0$

اما برهنة شروط الانحدار والانحدار الكافي وشرط الترافق فقد تمت في الجزء الثالث. وبرهنة التقارب الثامل

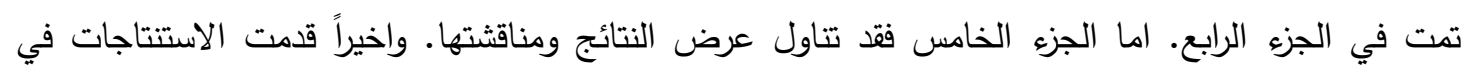

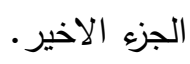

2- - خوارزمية الاتحدار المترافق الجديدة:

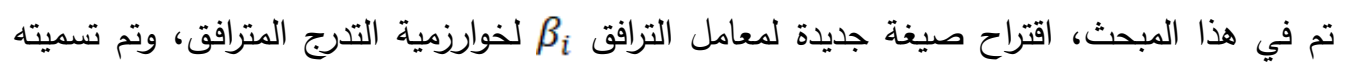
ب.

- اشتقاق الصيغة الجديدة لـ $t_{1}^{* *}=\frac{\left\langle v_{i}, y_{i>}\right.}{\left\|v_{i}\right\|^{2}}+\frac{\left\|y_{i}\right\|}{\left\|v_{i}\right\|}$ اقترح الباحثان Kafaki و Ghanbari قيمة جديدة لـ $t_{2}^{* *}=\frac{\left\|y_{i}\right\|}{\left\|v_{i}\right\|}$

وللمزيد من التفاصيل انظر [8]. والان، ناخذ التركيب الخطي : $t_{2}=\frac{\|y\|^{2}}{\left\|v_{i}\right\|^{2}}$ 
$t=\theta \frac{v_{i}^{T} y_{i}}{\left\|v_{i}\right\|^{2}}+(1-\theta) \frac{\|y\|^{2}}{\left\|v_{i}\right\|^{2}}$

$\beta_{i}^{N}=\frac{\left(g_{i+1}^{T}\left(y_{i}-\left(\theta \frac{v_{i}^{T} y_{i}}{\left\|v_{i}\right\|^{2}}+(1-\theta) \frac{\|y\|^{2}}{\left\|v_{i}\right\|^{2}}\right) v_{i}\right)\right.}{s_{i}^{T} y_{i}}$

وبالتعويض عن قيمة t في المعادلة (1.8)، نحصل على:

$\beta_{i}^{N}=\beta_{i}^{(H S)}-\theta \frac{v_{i}^{T} y_{i} g_{i+1}^{T} v_{i}}{s_{i}^{T} y_{i}\left\|v_{i}\right\|^{2}}-(1-\theta) \frac{\|y\|^{2} g_{i+1}^{T} v_{i}}{s_{i}^{T} y_{i}\left\|v_{i}\right\|^{2}}$

$$
\begin{aligned}
& \text { - الخوارزمية الجديدة لصيغة : } \\
& \text { الخطوة (1) : لتكن القيمة الابتدائية } \\
& \text { الخطوة (2) : لتكن } \\
& \text { الخطوة (3) : } \\
& \text { الخطوة (4) : : احسب قيمة } \alpha_{i} \text { لتقليل قيمة الدالة( } \\
& \text { الخطوة (5) : }
\end{aligned}
$$

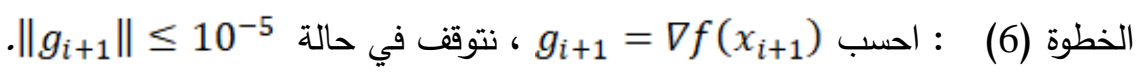

$$
\begin{aligned}
& \text { الخطوة (7) : : احسب قيمة (6) من المعادلة (2.4). } \\
& \text { الخطوة (8) : : احسب }
\end{aligned}
$$

الخطوة (9) : اذا تحققت

$$
\text { . } i=i+1
$$

3. شروط الانحدار والانحدار الكافي وشرط الترافق لخوارزمية الترافق الجديدة سنستعرض في هذا المبحث المبرهنات والنتائج التالية:

نظرية 3.1: نفرض ان المتسلسلة (1.6) و(1.7)، اذن قيمة

البرهان: من المعادلتين (1.3) و(1.4)، نحصل على:

$s_{i+1}=-g_{i+1}+\left(\beta_{i}^{(H S)}-\theta \frac{v_{i}^{T} y_{i} g_{i+1}^{T} v_{k}}{s_{i}^{T} y_{i}\left\|v_{i}\right\|^{2}}-(1-\theta) \frac{\|y\|^{2} g_{i+1}^{T} v_{i}}{s_{i}^{T} y_{i}\left\|v_{i}\right\|^{2}}\right) s_{i}$

$$
\text { وبضرب طرفي المعادلة اعلاه بـ }
$$

$g_{i+1}^{T} s_{i+1}=-\left\|g_{i+1}\right\|^{2}+\beta_{i}^{(H S)} g_{i+1}^{T} s_{i}-\theta \alpha_{i} \frac{v_{i}^{T} y_{i}\left(g_{i+1}^{T} s_{i}\right)^{2}}{s_{i}^{T} y_{i}\left\|v_{i}\right\|^{2}}-(1-\theta) \alpha_{i} \frac{\|y\|^{2}\left(g_{i+1}^{T} s_{i}\right)^{2}}{s_{i}^{T} y_{i}\left\|v_{i}\right\|^{2}}$

الحدين الاولين من المعادلة (3.2) تحققان شرط الانحدار (H/S)، أي أنه:

$-\left\|g_{i+1}\right\|^{2}+\frac{\left(g_{i+1}^{T} y_{k}\right)\left(g_{i+1}^{T} s_{i}\right)}{s_{i}^{T} y_{i}} \leq 0$

ومن الواضح أن قيم

الثالث والرابع موجبة. لذلك فإن الحدين الثالث والرابع من المعادلة (2.3) يكونان اقل او يساويان للصفر . 


$$
g_{i+1}^{T} s_{i+1}=-\left\|g_{i+1}\right\|^{2}+\beta_{i}^{H S} g_{i+1}^{T} s_{i}-\theta \alpha_{i} \frac{v_{i}^{T} y_{i}\left(g_{i+1}^{T} s_{i}\right)^{2}}{s_{i}^{T} y_{i}\left\|v_{i}\right\|^{2}}-(1-\theta) \alpha_{i} \frac{\|y\|^{2}\left(g_{i+1}^{T} s_{i}\right)^{2}}{s_{i}^{T} y_{i}\left\|v_{i}\right\|^{2}} \leq 0
$$

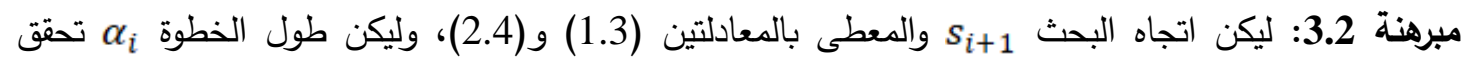
شروط ووالف Wolf القوية (1.4) و (1.5). فان المتباينة التالية:

$g_{i+1}^{T} s_{i+1} \leq-c\left\|g_{i+1}\right\|^{2}$

$i \geq 0$ مستمرة لكل قيم

البرهان:

من المبرهنة (1.3)، فان الحدود:

تكون اقل او تساوي الصفر ـ لذلك، نحتاج لبرهنة أن المعادلة التالية اقل او تساوي للصفر :

$g_{i+1}^{T} s_{i+1} \leq-\left((1-\theta) \alpha_{i} \frac{\|y\|^{2}\left(g_{i+1}^{T} s_{i}\right)^{2}}{s_{i}^{T} y_{i}\left\|v_{i}\right\|^{2}\left\|g_{i+1}\right\|^{2}}\right)\left\|g_{i+1}\right\|^{2}$

$$
c=(1-\theta) \alpha_{i} \frac{\|y\|^{2}\left(g_{i+1}^{T} s_{i}\right)^{2}}{s_{i}^{T} y_{i}\left\|v_{i}\right\|^{2}\left\|g_{i+1}\right\|^{2}}
$$

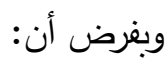

$g_{i+1}^{T} s_{i+1} \leq-c\left\|g_{i+1}\right\|^{2}$

فمن الواضح ان c> c c) لذا فان المعادلة (3.3) تصبح:

المبرهنة (3.3): اتجاه البحث s.1 المتولد من المعادلتين (1.3) و(2.4) يحقق شرط التوافق عندما يتم تحديد $\alpha_{i}$ بواسطة البحث في خط وولف (1.6) و (1.7)، أي أن:

$s_{i+1}^{T} y_{i}=-\tau g_{i+1}^{T} v_{i}$

إذ 0 إن

$s_{i+1}=-g_{i+1}+\beta_{i}^{N} s_{i}$

البرهان: من المعادلتين (1.3) و(2.4) نحصل على:

$$
\begin{aligned}
& \text { بضرب المعادلة اعلاه بـ } y_{i} \text { نحصل على: } \\
& s_{i+1}^{T} y_{i}=-g_{i+1}^{T} y_{i}+\frac{g_{i+1}^{T} y_{i}}{s_{i}^{T} y_{i}} s_{i}^{T} y_{i}-\theta \frac{v_{i}^{T} y_{i} g_{i+1}^{T} v_{i}}{s_{i}^{T} y_{i}\left\|v_{i}\right\|^{2}} s_{i}^{T} y_{i}-(1-\theta) \frac{\|y\|^{2} g_{i+1}^{T} v_{i}}{s_{i}^{T} y_{i}\left\|v_{i}\right\|^{2}} s_{i}^{T} y_{i}
\end{aligned}
$$

$s_{i+1}^{T} y_{i}=-\left(\theta \frac{v_{i}^{T} y_{i}}{\left\|v_{i}\right\|^{2}}+(1-\theta) \frac{\|y\|^{2}}{\left\|v_{i}\right\|^{2}}\right) g_{i+1}^{T} v_{i}$

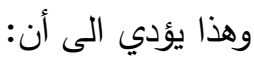

$\frac{v_{i}^{T} y_{i}}{\left\|v_{i}\right\|^{2}}+(1-\theta) \frac{\|y\|^{2}}{\left\|v_{i}\right\|^{2}}=\tau>0$

$s_{i+1}^{T} y_{i}=-\tau g_{i+1}^{T} v_{i}$ 
4. التقارب الثامل لخوارزمية التدرج المقترن الجديدة: في هذا المبحث، سنبرهن التقارب الشامل للطريقة الجديدة المقترحة.

- فرضية (H)

1. المجموعة

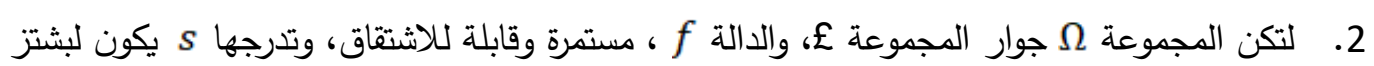

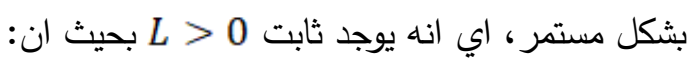

$\left\|g(x)-g\left(x_{i}\right)\right\| \leq L\left\|x-x_{i}\right\|, \forall x, x_{i} \in \Omega$

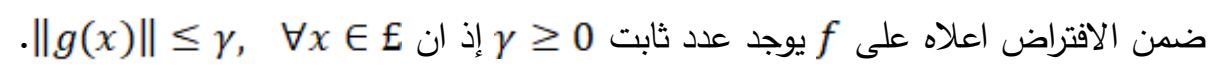

$\sum_{i \geqq 1} \frac{1}{\left\|s_{i}\right\|^{2}}=\infty$

نتيجة (4.1): ليكن الفرضية (H) متحققة عند اي تدرج المقترن (1.2) و (1.3)

$\lim _{i \rightarrow \infty} \inf \left\|g_{i}\right\|=0$,

$\lim _{i \rightarrow \infty}$ inf $\lg _{i} \|=0$,

اذا كانت f دالة محدبة بانتظام، اذاً يوجد عدد ثابت 0 > € بحيث ان:

$(g(x)-g(y))^{T}(x-y) \geq €\|x-y\|^{2} \in \Omega$

بالإمكان اعادة كتابة المعادلة (4.4) لتصبح بالصيغة التالية:

$y_{i}^{T} v_{i} \geq €\left\|v_{i}\right\|^{2}$

المبرهنة (4.1): لتكن الفرضية (H) متحققة وf دالة محدبة بانتظام، فان الخوارزمية الجديدة التي بالثكل

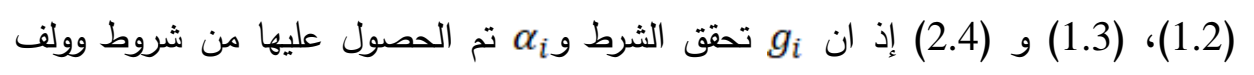

Wolf

$$
\begin{aligned}
& \lim _{i \rightarrow \infty} \text { inf }\left\|g_{i+1}\right\|=0 \\
& \text { البرهان: من المعادلتين (1.3) و (2.4) نحصل على: }
\end{aligned}
$$

$s_{i+1}=-g_{i+1}+\beta_{i}^{N} s_{i}$

$\left|\beta_{i}^{N}\right|=\left|\frac{g_{i+1}^{T} y_{i}}{s_{i}^{T} y_{i}}-\theta \frac{v_{i}^{T} y_{i} g_{i+1}^{T} v_{i}}{s_{i}^{T} y_{i}\left\|v_{i}\right\|^{2}}-(1-\theta) \frac{\|y\|^{2} g_{i+1}^{T} v_{i}}{s_{i}^{T} y_{i}\left\|v_{i}\right\|^{2}}\right|$,

$\Rightarrow\left|\beta_{i}^{N}\right| \leq\left|\frac{g_{i+1}^{T} y_{i}}{s_{i}^{T} y_{i}}\right|+\left|\theta \frac{v_{i}^{T} y_{i} g_{i+1}^{T} v_{i}}{s_{i}^{T} y_{i}\left\|v_{i}\right\|^{2}}\right|+\left|(1-\theta) \frac{\|y\|^{2} g_{i+1}^{T} v_{i}}{s_{i}^{T} y_{i}\left\|v_{i}\right\|^{2}}\right|$,

$g_{i+1}^{T} v_{i} \leq \alpha_{i} s_{i}^{T} y_{i}:$ وبما

$\therefore\left|\beta_{i}^{N}\right| \leq\left|\frac{g_{i+1}^{T} y_{i}}{s_{i}^{T} y_{i}}\right|+\left|\theta \alpha_{i} \frac{v_{i}^{T} y_{i}}{\left\|v_{i}\right\|^{2}}\right|+\left|(1-\theta) \frac{\|y\|^{2} g_{i+1}^{T} v_{i}}{s_{i}^{T} y_{i}\left\|v_{i}\right\|^{2}}\right|$,

$$
\begin{aligned}
€\left\|v_{i}\right\|^{2} \leq y_{i}^{T} v_{i} & \text { من المعادلة (4.5) }
\end{aligned}
$$

وبما ان: ||

$\left|\beta_{i}^{N}\right| \leq \frac{\alpha_{i} L\left\|g_{i+1}\right\|}{€\left\|v_{i}\right\|}+\theta \alpha_{i} L+(1-\theta) \alpha_{i} L^{2}$, 


$$
\begin{aligned}
& \left|\beta_{i}^{N}\right| \leq \frac{\alpha_{i} L \gamma}{€\left\|v_{i}\right\|}+\theta \alpha_{i} L+(1-\theta) \alpha_{i} L^{2}, \\
& \left\|s_{i+1}\right\| \leq\left\|g_{i+1}\right\|+\left|\beta_{i}^{N}\right|\left\|s_{i}\right\|, \\
& \left\|s_{i+1}\right\| \leq \gamma+\left(\frac{\alpha_{i} L \gamma}{€\left\|v_{i}\right\|}+\theta \alpha_{i} L+(1-\theta) \alpha_{i} L^{2}\right)\left\|s_{i}\right\|, \\
& \left\|s_{i+1}\right\| \leq \gamma+\left(\frac{L \gamma}{€\left\|v_{i}\right\|}+\theta L+(1-\theta) L^{2}\left\|v_{i}\right\|\right) . \\
& \quad\left\|v_{i}\right\|=\left\|x-x_{i}\right\|, \\
& \left.D=\max \left\{\left\|x-x_{i}\right\|\right\}, \forall x, x_{i} \in R\right\} \\
& \left\|s_{i+1}\right\| \leq \gamma+\left(\frac{L \gamma}{€ D e s}+\theta L+h(1-\theta) L^{2} D e s\right)=\varphi . \\
& \sum_{i \geqq 1} \frac{1}{\left\|s_{i+1}\right\|^{2}} \geq \sum_{i \geqq 1} \frac{1}{\varphi^{2}}=\sum_{i \geqq 1} 1=\infty \\
& \Rightarrow \sum_{k \geqq 1} \frac{1}{\left\|s_{k+1}\right\|^{2}}=\infty .
\end{aligned}
$$$$
\text { وهذا يؤدي الى انه: }
$$

وبالتالي المعادلة (4.12) ستصبح

$\lim _{i \rightarrow \infty}$ inf $\left\|g_{i+1}\right\|=0$

تضمنت اختبارات المقارنة (دوال الاختبار الاصلية) ذات أبعاد مختلفة تتراوح بين 45 اعتماد لغة الفورترن 95 لبرمجة الخوارزمية الجديدة، وباستعمال شرط التوقف لكل في الاصليهات الحالات 年 $\left|g_{i}^{T} g_{i+1}\right| \geq 0.2\left\|g_{i+1}\right\|^{2}$ والروتين الفرعي للبحث الخطي هو الاستكمال التكعيبي الذي يستعمل قيم الدالة والانحدار.

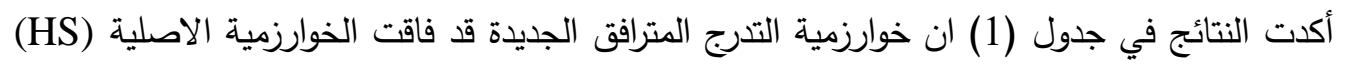
فيما يتعلق بعدد التكرارات الكلي NOI وعدد قيم الدوال الكلي NOF. 
الجدول (1): مقارنة أداء الذوارزميتين الاصلية (HS) والجديدة

\begin{tabular}{|c|c|c|c|c|c|}
\hline \multicolumn{2}{|c|}{ الخوارزمية الجديدة } & \multicolumn{2}{|c|}{ الخوارزمية الاصلية H/S } & \multirow[b]{2}{*}{ عدد المتغيرات } & \multirow[b]{2}{*}{ المسائل } \\
\hline $\begin{array}{c}\text { عدد قيم الدوال الكلي } \\
\text { NOF }\end{array}$ & $\begin{array}{c}\text { عدد التكرارات الكلي } \\
\text { NOI }\end{array}$ & $\begin{array}{c}\text { عدد قيم الدوال الكلي } \\
\text { NOF }\end{array}$ & $\begin{array}{c}\text { عدد التكرارات الكلي } \\
\text { NOI }\end{array}$ & & \\
\hline 61 & 27 & 68 & 30 & 4 & \\
\hline 61 & 27 & 68 & 30 & 100 & \\
\hline 61 & 27 & 68 & 30 & 500 & 1 \\
\hline 61 & 27 & 68 & 30 & 1000 & \\
\hline 61 & 27 & 68 & 30 & 5000 & \\
\hline 147 & 21 & 159 & 22 & 4 & \\
\hline 158 & 22 & 159 & 22 & 100 & \\
\hline 158 & 22 & 171 & 23 & 500 & 2 \\
\hline 171 & 23 & 171 & 23 & 1000 & \\
\hline 171 & 23 & 248 & 28 & 5000 & \\
\hline 64 & 24 & 64 & 24 & 4 & \\
\hline 79 & 29 & 79 & 29 & 100 & \\
\hline 80 & 30 & $\mathrm{~F}$ & $\mathrm{~F}$ & 500 & 3 \\
\hline 80 & 30 & 79 & 29 & 1000 & \\
\hline 80 & 30 & 81 & 30 & 5000 & \\
\hline 87 & 28 & 85 & 28 & 4 & \\
\hline 104 & 31 & 114 & 33 & 100 & \\
\hline 139 & 38 & 146 & 40 & 500 & 4 \\
\hline 139 & 38 & 176 & 46 & 1000 & \\
\hline 139 & 38 & 211 & 54 & 5000 & \\
\hline 67 & 27 & 108 & 38 & 4 & \\
\hline 85 & 33 & 122 & 40 & 100 & \\
\hline 85 & 33 & 124 & 41 & 500 & 5 \\
\hline 85 & 33 & 124 & 41 & 1000 & \\
\hline 102 & 36 & 124 & 41 & 5000 & \\
\hline 27 & 5 & 11 & 3 & 4 & \\
\hline 80 & 14 & 81 & 14 & 100 & \\
\hline 120 & 21 & 124 & 21 & 500 & 6 \\
\hline 109 & 22 & 128 & 23 & 1000 & \\
\hline 156 & 31 & 159 & 31 & 5000 & \\
\hline 24 & 11 & 24 & 11 & 4 & \\
\hline 89 & 44 & 99 & 49 & 100 & \\
\hline 91 & 45 & 105 & 52 & 500 & 7 \\
\hline 107 & 53 & 141 & 70 & 1000 & \\
\hline 312 & 150 & 348 & 165 & 5000 & \\
\hline 3640 & 1120 & 4225 & 1281 & & \\
\hline
\end{tabular}

الجدول (2): مقارنة معدل التحسن بين الخوارزميتين الاصلية HS والجديدة

\begin{tabular}{|c|c|c|}
\hline الخوارزمية الجديدة & الخوارزمية الاصلية HS & الاداة \\
\hline $87.4317 \%$ & $100 \%$ & عدد التكر ار ات الكلي N.O.I. \\
\hline $86.1538 \%$ & $100 \%$ & Nلعد قيم الدو ال الكلي N.O.F. \\
\hline
\end{tabular}


أظهرت النتائج في الجدول (2) معدل التحسن في الخوارزمية الجديدة مقارنة بالخوارزمية الاصلية HS. اذ كانت نتائج الخوارزمية الجديدة افضل من الخوارزمية الاصلية.

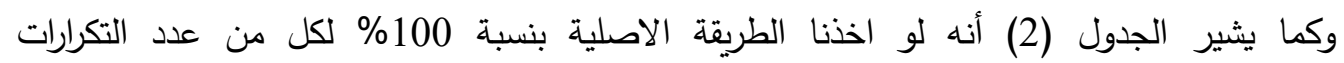
الكلي NOI وعدد قيم الدوال الكلي NOF، وهذا يعني أن الخوارزمية الجديدة تحسنت مقارنة مع الخوارزمية الاصلية بنسبة 12.56٪ في NOI و 13.84\% في NOF. وبشكل عام، فان الخوارزمية الجديدة تحسنت بنسبة 13.2073\% مقارنة مع خوارزمية (HS) الاصلية.

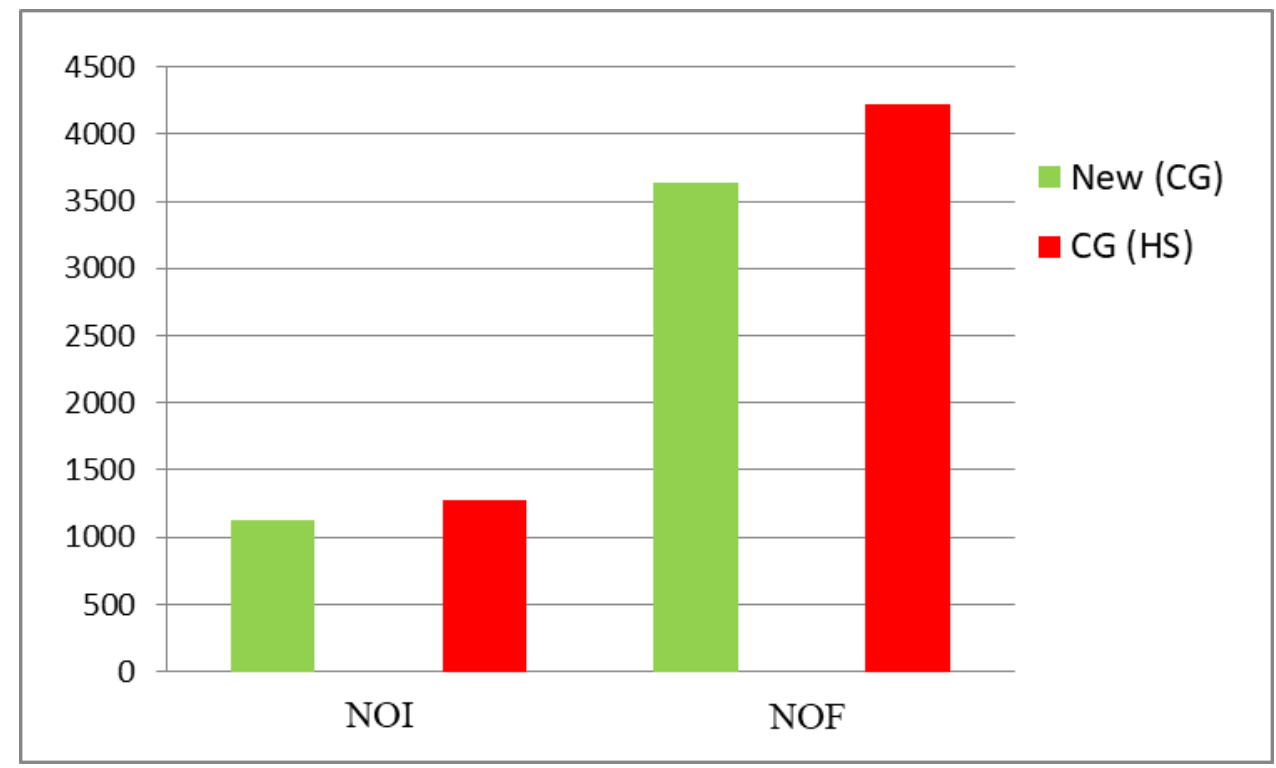

NOI الثكل (1): يبين المقارنة بين الخوارزمية الجليدة والخوارزمية الأصلية (HS) وفقًا لإجمالي عدد التكرارات الكلي NOF. وعدد قيم الدوال الكلي

الصيغة الجديدة التي تم اقتراحها لطريقة التدرج المترافقة التي تعتمد على صيغة داي-لياو (DL) لحل مسائل الامثلية غير المقيدة والتي تعتمد على صيغة كافاكي و غانبري هي صيغة جيدة مقارنة بمثيلاتها في هذا المجال وقد تم بيان ذلك من خلال المقارنة عدديا مع خوارزميات اخرى حيث اظهرت النتائج افضلية الخوارزمية الجديدة على خوارزمية HS في عدد التكرارت NOI وعدد حساب الدالة NOF. ميككن ان نستخدم طريقة اخرى في المستقبل لإيجاد قيمة t في صيغة DL وبالتالي الحصول على خوارزميات جديدة. 


\section{Reference}

[1] Dai, Y. H. and Liao, L.Z., (2001), New conjugacy conditions and related nonlinear conjugate gradient methods, Application Mathematical Optimization, 43, 87-101.

[2] Dai, Y. H. and Yuan, Y. (1999), A nonlinear conjugate gradient method with a strong global convergence property, SIAM Journal on Optimization, 10 , 177 182.

[3] Dai, Y. H. and Yuan, Y., (1996), Convergence properties of the FletcherReeves method, IMAJ. Numer. Anal., 2, 155-164.

[4] Fletcher, R. and Reeves, C.M., (1964), Function minimization by conjugate gradients, The Computer Journal. 7, 149-154.

[5] Fletcher, R., (1987), Practical methods of optimization unconstrained optimization, John Wiley \& Sons, New York, NY, USA.

[6] Hager, W. W. and Zhang, H., (2006), A survey of nonlinear conjugate gradient methods, Pacific Journal of Optimization, 2, 35-58.

[7] Hestenes, M. R. and Stiefel, E., (1952), Methods of conjugate gradients for solving linear systems, Journal of Research of the National Bureau of Standards. 49, 409-436.

[8] Kafaki, S. B. and Ghanbari, R., (2014), The Dai-Liao nonlinear conjugate gradient method with optimal parameter choices, European Journal of Operational Research, 234, 625-630.

[9] Liu, Y. and Storey, C., (1991), Efficient generalized conjugate gradient algorithms, part 1: Theory, Journal of Optimization Theory and Applications, 69, 129-137.

[10] Nocedal, J. and Wright, S.J., (2006), Numerical optimization (Second Edition), Springer Series in Operation Research, Springer Verlag, New York.

[11] Polak, E. and Ribiere, G., (1969), Note surla convergence des méthodes de directions conjuguées., 3(16), 35-43.

[12] Polyak, B. T., (1969), The conjugate gradient method in extreme problems, USSR Comp. Math. and Math. Phys., 94-112.

[13] Wolfe, P. (1969), Convergence conditions for ascent methods, SIAM. Rev. 11, 226-235.

[14] Zoutendijk, G., (1970), Nonlinear Programming, Computational Methods, in: J. Abadie (Ed.), Integer and Nonlinear Programming, North-Holland. 


\section{الملحق}

اختبار دالة الامثلية غير المقيدة

$$
\begin{gathered}
\text { [ } f(x)=\sum_{i=1}^{n / 4}\left(100\left(x_{4 i-3}^{2}-x_{4 i-2}\right)^{2}+\left(x_{4 i-3}-1 .\right)^{2}+90\left(x_{4 i-1}^{2}-x_{4 i}\right)^{2}\right. \\
\quad+\left(1 .-x_{4 i-1}\right)^{2}+10.1\left(x_{4 i-2}-1 .\right)^{2}+\left(x_{4 i}-1 .\right)^{2} \\
\left.\quad+19.8\left(x_{4 i-2}-1 .\right)\left(x_{4 i}-1 .\right)\right), \\
\left.x_{0}=(-3,-1, \ldots,-3,-1)^{T} . .\right]
\end{gathered}
$$

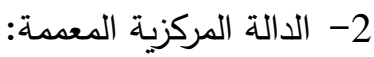

$$
\begin{aligned}
& {\left[\quad f(x)=\sum_{i=1}^{n / 4}\left(\exp \left(x_{4 i-3 .}+x_{4 i-2 .}\right)^{4}+100 .\left(\left(x_{4 i-2}-x_{4 i-1 .}\right)^{6}\right.\right.\right.} \\
& +\arctan \left(\left(x_{4 i-1}-x_{4 i}\right)^{4}+x_{4 i-3}\right) \text {, } \\
& x_{0}=(1,2,2,2, \ldots, 1,2,2,2)^{T} . . \quad \text { ] }
\end{aligned}
$$

3- الدالة غير القطرية المعمدة:

$\left[\quad F(x)=\sum_{i=2}^{n}\left(100 .\left(x_{1}-x_{i}^{2}\right)^{2}+\left(1-x_{i}\right)^{2}\right), x_{0}(-1, \ldots,-1)^{T} . . \quad\right.$ ]

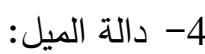

$$
\begin{aligned}
{[\quad F(x)=} & \sum_{i=1}^{n / 4}\left(\left(e^{x_{4 i-3}}+10 x_{4 i-2 .}\right)^{2}+100\left(x_{4 i-2}+x_{4 i-1}\right)^{6}+\left(\tan \left(x_{4 i-1}-x_{4 i}\right)\right)^{4}\right. \\
& \left.+\left(x_{4 i-3}\right)^{8}+\left(x_{4 i}-1 .\right)^{2}\right) \\
& \left.x_{0}=(1,2,2, \ldots, 1,2,2)^{T} . .\right]
\end{aligned}
$$

5- 5 - 5 دالة باول:

$$
\begin{gathered}
{\left[\quad F(x)=\sum_{i=1}^{n / 4}\left(\left(x_{4 i-3}-10 x_{4 i-2 \cdot}\right)^{2}+5\left(x_{4 i-1}-x_{4 i}\right)^{2}+\left(x_{4 i-2}-2 x_{4 i-1}\right)^{4}\right.\right.} \\
\left.\quad+10\left(x_{4 i-3}-x_{4 i}\right)^{4}\right), x_{0}=(3,-1,0,1, \ldots, 3,-1,0,1)^{T} . .
\end{gathered}
$$

$\left[F(x)=\sum_{i=1}^{n}\left(x_{i}-i\right)^{4}, x_{0}=(1,1, \ldots, 1)^{T}.\right]$

[ $\quad F(x)=\left(-x_{1}\left(3 .-\frac{x_{1}}{2}\right)+2 \cdot x_{2}-1 .\right)^{2}$

$$
\begin{aligned}
& +\sum_{i=1}^{n-1}\left(x_{i-1 .}-x_{i}\left(3 .-\frac{x_{i}}{2 .}+2 . x_{i+1}-1 .\right)\right)^{2} \\
& \left.+\left(x_{n-1}-x_{n}\left(3 .-\frac{x_{n}}{2 .}\right)-1 .\right)^{2}, x_{0}=(-1, \ldots,-1)^{T} . . \quad\right]
\end{aligned}
$$

